\title{
Air pollution and fog detection through vehicular sensors
}

\author{
Clemens Dannheim ${ }^{1}$, Christian Icking ${ }^{2}$, Markus Mäder ${ }^{1}$, Philip Sallis ${ }^{3}$, Vladimir Fisher ${ }^{1}$ \\ ${ }^{1}$ Objective Software GmbH, ${ }^{2}$ FernUniversität Hagen, ${ }^{3}$ Auckland University of Technology \\ \{clemens.dannheim, markus.maeder\}@objective.de, christian.icking@ fernuni-hagen.de, philip.sallis@aut.ac.nz
}

\begin{abstract}
We describe a method for the automatic recognition of air pollution and fog from a vehicle. Our system consists of sensors to acquire main data from cameras as well as from Light Detection and Recognition (LIDAR) instruments. We discuss how this data can be collected, analyzed and merged to determine the degree of air pollution or fog. Such data is essentaial for control systems of moving vehicles in making autonomous decisions for avoidance. Backend systems need such data for forecasting and stragtegic traffic planning and control. Laboratory based experimental results are presented for weather conditions like air pollution and fog, showing that the recognition scenario works with better than adequate results. This paper demonstrates that LIDAR technology, already onboard for the purpose of autonomous driving, can be used to improve weather condition recognition when compared with a camera only system. We conclude that the combination of a front camera and a LID AR laser scanner is well suited as a sensor instrument set for air pollution and fog recognition that can contribute accurate data to driving assistance and weather alerting systems.
\end{abstract}

Keywords: air pollution detection; fog detection; weather detection; remote sensing; LIDAR; colaborative driver assistant functions; spatial resolution; air pollution forecasting services

\section{INTRODUCTION}

Driving assistance functions are core technologies of intelligent vehicles. Well-known applications are lane keeping, traffic sign detection and interpretation, light assistance and adaptive cruise control. All of those functions are capable of scanning visual images and to determine lanes, signals and lights of preceding or opposing traffic. Recognising the extent of air pollution in the proximity of a traveling vehicle such as fog and aerosol particulate matter density, is a real challenge and can provide important auxiliary information for many other driving applications considering the degree of air pollution. The spectrum of air pollution and fog condition information obtained by this combination of instruments is categorised with both quantitative and qualitative methods.

LIDA R technology enables advanced object recognition, used for more qualified detection in quality and quantity (fog, dust, drizzle, mist, aerosol). LIDAR is a promising technology for air pollution and fog detection, due to its very high sensitivity and spatial resolution, this active detection technique enables efficient location of air pollution and weather effects during both day and night and over a considerable range.

The fusion of camera and LIDAR air pollution with fog condition data provides valuable input for generating reliable and robust information on certain pollution, vision and weather effects. This is described here in the paper.

Finally, a neural- network technique for automatic recognition of severe air pollution and fog signatures on incoming camera and LIDAR data is considered and several neural-network architectures and learning algorithms are evaluated and presented.

\section{CAMERA BASED WEATHER RECOGNITION}

Vehicle on-board mounted cameras capture the visual signal in which the driver needs to drive safely. Processing this in real time allows detecting pavement markings, road signs or hazards such as obstacles, in order to as ses s time to lane crossing, time to collision or other risk indicators. It is also possible to detect reduced visibility conditions such as fog, rain, or glare, and even to quantify their impact on sight distance or target visibility, in order to provide speed warning, distance warning to vehicles ahead or to control light beams. In some cases, visibility can even be restored, up to a point, by dehazing and in-painting techniques for Head-Up-Dis plays [2].

\section{A. Detection and characterization of adverse weather conditions}

Contemporary modern cameras are widely-used and are a component of almost every advance driver assistance system because of the their price and flexibility. Nevertheless the major problem is that cameras can be blind under adverse weather conditions when they are needed the most. To identify and quantify the cause of the degradation of the signal and estimate a confidence index is a strong challenge for ADAS development.

\section{B. Detection of rain, snow and fog}

Previous camera based fog detection systems analyse distinct objects in the image or image regions like the road region or the horizon. These approaches are not reliable for everyday use. A new method is to use the only reliable visible attribute of foggy weather conditions: the decrease in contrast and blurring in the whole image [1]. The power spectrum being the squared magnitude of the Fourier transform for the image that holds information about the frequencies in the image. The matter of discarding spatial information and its decision-informing potential will be analysed. From the power spectrum one builds the image features that can be then fed to the classifier that has been trained on fog and fog free images. It turns out, that in the case of an observed fog scene, the frequency components are concentrated at the zero frequency whereas in a scene 
without fog one finds a broadly spread spectrum caused by the contrast attenuation and blurring in the image provoked by the fog. Sharp Edges are modelled by many different low and high frequencies, where as smooth edges created by only low frequencies.

The key to a single step fog detection is now to work out the differences in the power spectrum.[1]

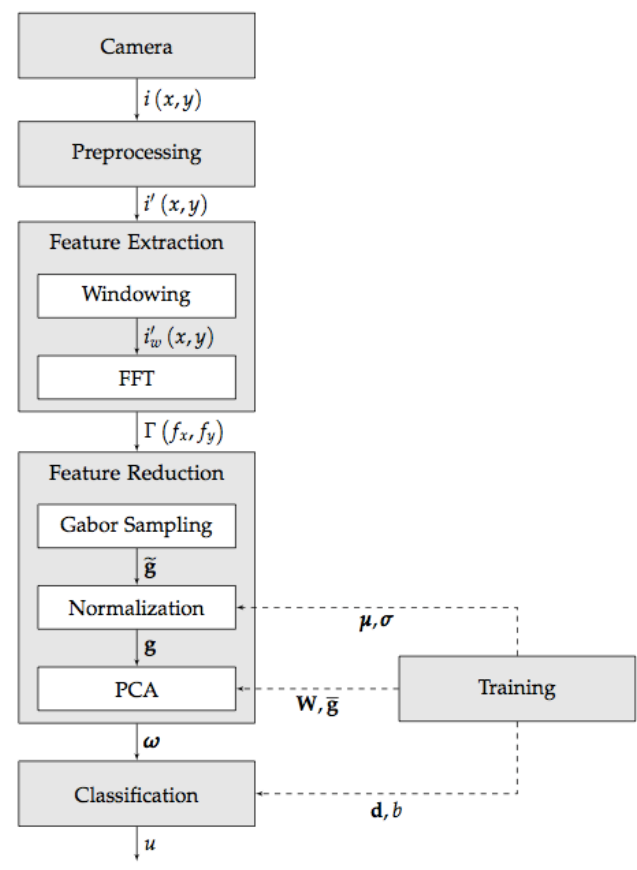

Fig. 1 Fog detection flow diagram

In the preprocessing step the image will be filtered and normalized to reduce illumination effects and to avoid that some image regions dominate the spectrum. For the normalization a square image section is used and the intensity values are equalized.

$$
i^{\prime}(x, y)=\frac{i(x, y) * h(x, y)}{\varepsilon+\sqrt{[i(x, y) * h(x, y)]^{2} * g(x, y)}}
$$

Next step is the feature extraction. Before the Fourier transformation a windowing with a 2 dimensional Hanning window is applied [1].

$$
\Gamma(f x, f y)=\left|\sum_{x=0}^{N-1} \sum_{y=0}^{N-1} i^{\prime}(x, y) e^{-j 2 \pi\left(f_{x} x+f y\right.}\right|
$$

For the feature reduction a Gabor sampling by a filter bank of scaled and oriented filters is deployed. For the subsequent PCA the Gabor features are used.

$$
G_{i}\left(f_{x}, f_{y}\right)=e^{-2 \pi^{2}\left(\sigma_{x}^{2}\left(f^{\prime}{ }_{x}-f_{r}\right)^{2}+\sigma_{y}^{2} f_{y}^{2}\right)}, i=1, \ldots K
$$

In the last step of the Classification Process, a SVM (Support Vector Machine) with a Radial Basis Function (RBF) kernel is used. The regularization parameters were determined by training data.

With this approach summarized above, the average accuracy accomplished was $96 \%$ on a basis of 44.000 images [1]. The next step would be to analyze how this method would perform on nighttime fog images. At night time light from the headlamps is reflected by the airborne droplets of the fog in front of the vehicle back to the driver, producing a back-scattered veil. This would create a blurring effect as encountered during daytime. Our research is investigating the potential for this by extending the method to further distinguish rain and snow from fog.

\section{LIDAR BASED AIR POLLUTION \& FOG RECOGNITION}

\section{A. Detection and characterization of adverse weather conditions}

Despite their price and versatility, cameras have their disadvantages. One major problem is that cameras can be totally or partially blind under adverse weather conditions, when the systems they feed are most needed. Detecting and quantifying degradations of the signal and identify ing their causes constitutes an as yet unresolved challenge for automated driver assistance systems (ADAS). It involves estimating a confidence index, and it involves selfdiagnosis. Another sensor technology is LIDAR (Light Detection and Ranging). It is based on laser technology and eliminates most of the common disadvantages of cameras.

\section{$B$. The LIDAR priciples and pulse reflection recognition}

The principle behind LIDAR is quite simple. A narrow light beam is shone at a surface and the time it takes to return to its source is measured. This is similar to when one shines a torch light onto a surface and what is actually seen is the light being reflected and returned to the retina of the viewer. Light travels very fast - about 300,000 kilometres per second or 0.3 metres per nanosecond. The equipment required to carry out this operation needs therefore, to operate extremely fast. Only with the advancements in modern computing technology has this instrumentation operation become feasible.

The actual calculation used to measure how far a returning light photon has travelled to and from an object is quite simple:

$$
\text { Distance }=\frac{(\text { Speed of light } * \text { Time of flight })}{2}
$$

The LIDAR sends rapid pulses of laser light at a surface (fig. 2, 4), some at up to 150,000 pulses per second. A sensor on the device measures the amount of time (fig. 2, 3) it takes for each pulse to bounce back (fig. 2, 1). Light moves at a constant and known speed so the LIDAR can calculate the distance between itself and the target with high accuracy. By repeating this in quick succession the device builds up a complex 'map' of the surface scanned. 




Fig. 2: 1 Object, 2 Laser pulse 3 Reflected laser pulse 4 Laser

Lasers are categorised by their wavelength. $600-1000 \mathrm{~nm}$ lasers are more commonly used for non-scientific purposes but, as they can be focused and easily absorbed by the eye, the maximum power has to be limited to make them 'eyesafe'. Lasers with a wavelength of $1550 \mathrm{~nm}$ are a common alternative as they are not focused by the eye and are 'eyesafe' and can be used at higher power levels [9].

For a LIDAR sensor mounted on vehicles, it is necessary to determine the absolute position and the orientation of the sensor to retain useable data. Global Positioning Systems provide accurate geographical information regarding the position of the sensor and an Inertia Measurement Unit (IMU) records the precise orientation of the sensor at that location. These two devices provide the method for translating sensor data into static points for use in a variety of systems.

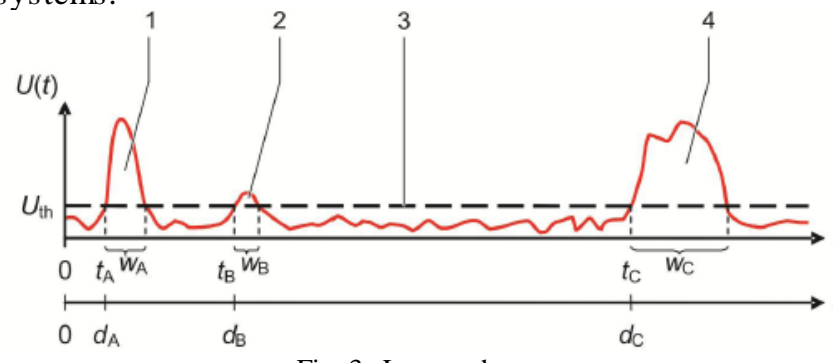

Fig. 3: Laser echo $\begin{array}{ll}1 & \text { Reflecion windshield } \\ 2 & \text { Reflecion rain drop } \\ 3 & \text { Threshold Voltage Uth } \\ 4 & \text { Object reflecion } \\ \text { C Object }\end{array}$

LIDAR is becoming more important as an environmental recognition sensor for highly automated vehicles. The speed and accuracy of a scanner enables applications of it to operate in more or less real-time. This enables the device to detect obstacles and to recognise its route in very small time intervals. This means that the near range weather situation around a vehicle can be captured.

Within fig. 3 the pulse 4 indicates the reflection of an object on the trajectory of a vehicle in a certain sensible distance. The voltage Uth indicates the noise threshold, at signal levels above the threshold, so indicating an object reflection.

\section{Object detection out of the pulse reflection cloud}

For the research vehicle the LIDAR system IBEO-LUX has been used. The system produces as output a stream of range and angle tuples; this data is projected into the local coordinate system using the vehicle's position in the local coordinate system (continuously updated as the vehicle moves) and the sensor's position in the vehicle's coordinate system (determined off-line). The result is a stream of 3D points in the local coordinate frame (fig. 4), where all subsequent sens or fusion takes place.

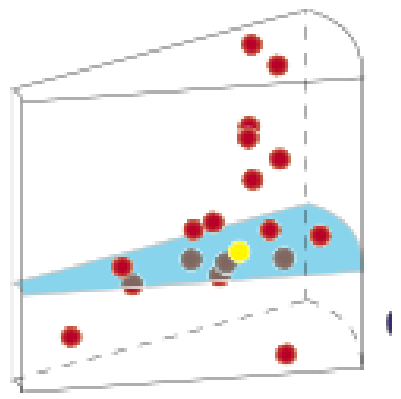

Fig. 4: Scanned weather objects in a polar grid

The LIDAR returns detected objects marked as ground, dirt, rain, snow, spray and fog. The first phase of the data processing is to classify each return as "ground", "dirt", or "rain/spray/fog". The first step is to filter out the stream of points that correspond only to weather objects (removing ground and outliers). By accomplishing this step, the high resolution of LIDAR data enables us to implement a sophisticated weather classifier. This is essential for our work, which set out to develop a strategy for identifying objects that are likely to be remarkable weather objects; those of immediate importance for driving condition recognition. Other points in the cloud that are far enough above the ground model (and satisfy other criteria designed to reject outliers) are output as weather detections.

Particle filters such as Kalman or Gaborfilters have been the subject of research and verification processes in our project. They are used to classify and quantity the examined weather type objects. Given the non-conformity of the airborne moisture laden entities being detected it is suggested that allometric scaling algorithms may be useful for analysis of cloud/fog/rain/snow/hail/haze and mist entities, especially from $\mathrm{v}$ isual onboard instruments located in moving vehicles. These are being investigated for inclusion in the set of techniques potentially applicable to the domain of research being described in this paper. Not only topographic characterization for entity shape is applicable in this domain, with LIDAR bathymetric methods also being considered for the useful provision of detected moisture content.

Both of these geometric recognition and characterization methods are to be the subject of ongoing investigation in the work.

\section{Detection of rain, snow and fog}

During weather effects such as fog, rain, and snow, the backscatter leads to the perception of an atmospheric veil. Therefore, we look at the LIDAR reflected signal power (Form. 2) and the backscatter coefficient $\langle\beta(R)\rangle$. 
The power $P_{r}$ received by the LIDAR is defined by the LIDAR equation (Measures, 1984)

$$
P_{r}(R)=E_{l} \frac{c\langle\beta(R)\rangle}{2} \frac{A_{r}}{R^{2}} \tau_{T} \tau_{R} \exp \left(-2 \int_{0}^{R} \alpha\left(R^{\prime}\right) d R^{\prime}\right)
$$

where $\mathrm{El}$ is the output laser pulse energy, $\mathrm{c}$ the speed of light, $\beta(R)$ the mean backscattering coefficient of the medium, $A_{r}$ the effective receiver area, $\tau_{\mathrm{T}}$ and $\tau_{\mathrm{R}}$ the transmitter and receiver efficiencies (the latter is mostly defined by a special filter confining the bandwidth), and $\alpha$ is the extinction coefficient [4].

The backscattering coefficient $\langle\beta(R)\rangle$ is an average over the area illuminated by the laser beam as well as along the line-of-sight within the range $c t_{p} / 2$, where $t_{p}$ is the laser pulse duration. The extinction coefficient $\alpha$ is controlled by the aerosolscattering fact.

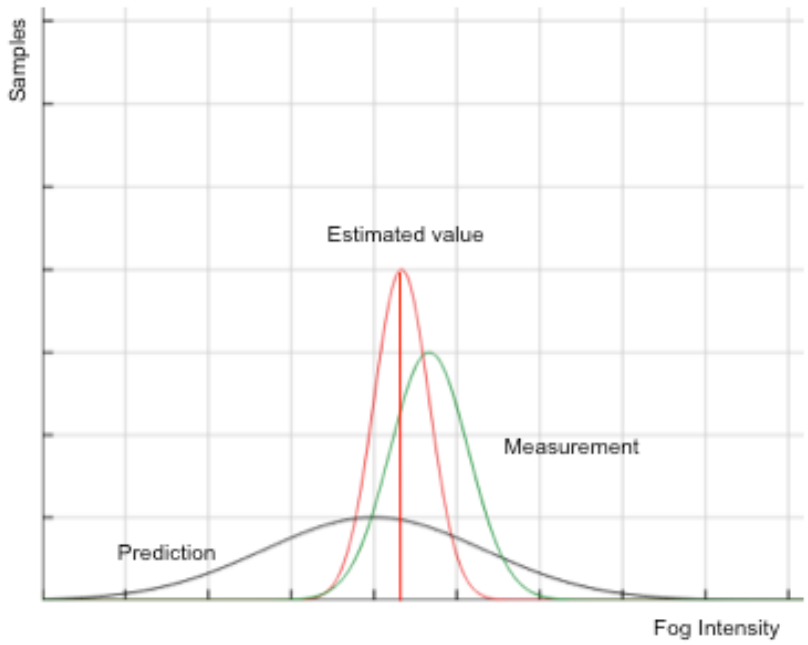

Fig. 5 Parameter intensity

For object and obstacle detection for adaptive cruise control (ACC) or highly automated driving the weather influence is filtered out (resulting as backscatter and power of the received LIDAR reflection). For obtaining qualified weather object information the LIDAR reflections have to be particle filtered via Kalman filters. A particular focus is put on the backscatter coefficient $\beta$ for variation of the filter.

Estimates for fog intensity are statistically calculated using a Kalman filter algorithm in an ensemble of error correcting procedures to produce a most probable estimate value for fog intensity. This method has become known as the Ensemble Kalman Filter $(E n K F)$ method. An estimate value is provided to the algorith $m$ as an initial prior that is sensitized by intervention values from the sensor data to provide a posterior value, which recursively becomes the new prior (estimate) for the next iteration of the filter. The Kalman filter correlates the historic data of previous sample data with the value of the new prior, producing an error value (the corrected most probable value), which becomes the estimate, or estimated intensity value as illustrated in fig. 5 .

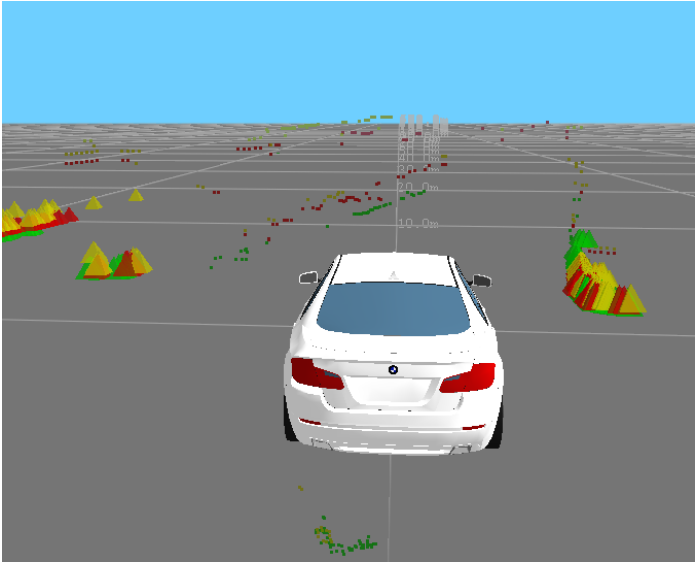

Fig. 6 Detection Scenario

We examine a set of filters under lab conditions to determine weather condition information from the vehicle's trajectory when in a $200 \mathrm{~m}$ range of known objects. The set of weather condition data comprises:

- Rain

- Snow

- Fog, Dust, Mist, Haze

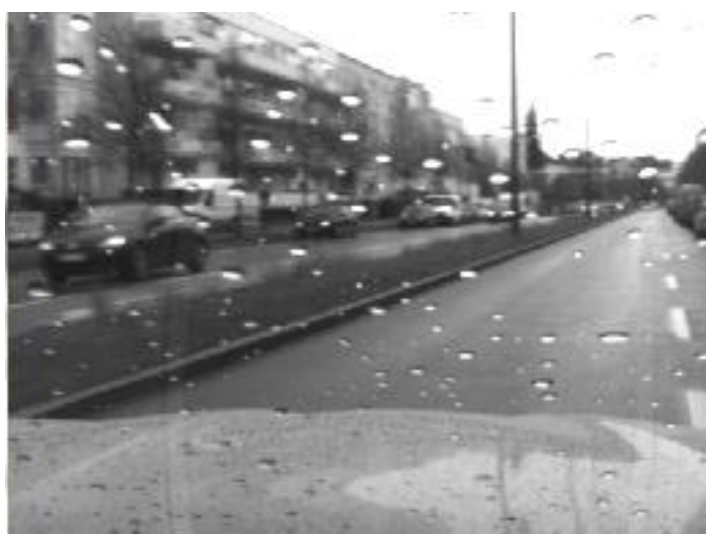

Fig. 7 Front camera

At fig. 6 you can see the LIDAR based rain detection scenario on the research vehicle. The associated camera image is illustrated within fig. 7. The object and obstacle detection is outlined. As result of the weather depiction, the LIDA R point cloud around the rain object recognition can be seen. A Kalman filter was utilized throughout the implementation. The pseudo code at fig. 8 visualizes the parameterization for the weather detection purposes, fig. 9 reflects the stochastic calculation. 


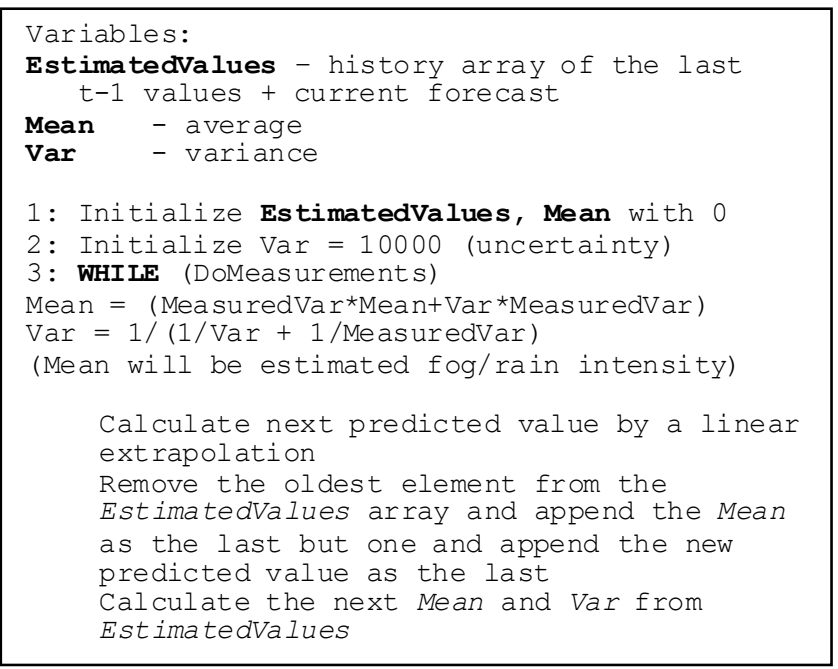

Fig. 8 Kalman filter settings

\section{SENSOR FUSION, DATAFUSION}

"Sensor fusion is a formal framework that comprises methods and tools for the association of data coming from different sensory sources. It tries to win information of high quality, where the specific definition of high quality varies also from one application to another" (Wald).

"Data fusion is the process of combining data or information to estimate or predict entity states" (Steinberg).

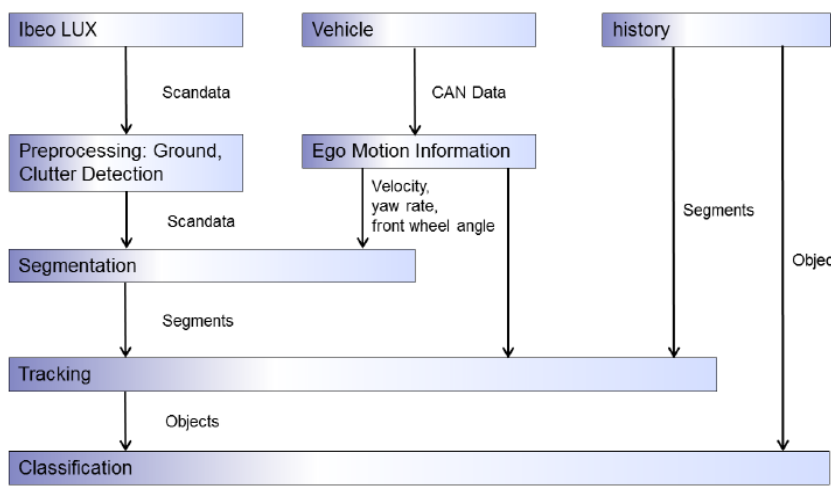

Fig. 9 Sensor fusion for gaining weather information

As described earlier within the document, different sensors are delivering data for ACC or automated driving. Discussion of a lane keeping function demonstrates that there are essential sensors needed for this such as

- Camera

- LIDAR

- Position (GPS)

- Velocity

- Inertia Measurement Unit (IMA)

in order to obtain sufficient information about the specific position and movement needed to control the vehicle on its planned trajectory. To combine this spectrum of sensor data, a fusion model is introduced (see fig. 8). Vertical segmentation covers the different sensor types and sources, horizontal segmentation is done for functional composition and logical grouping.

In case of air pollution and fog recognition using LIDAR, camera and ego, motion is a primary value used in the air pollution and fog perception equation. Historical data of these sources are needed for considering the time variable element for the equation. The perception model developed to deliver precise environmental information needs historical data for tendency handling.

Fig. 10 illustrates the sensor data fusion process for LIDAR sensor data generation (data collection) through a process that determines the quality and quantity of that data. These so-called reflections are visualized. The final applicable LIDAR point clouds appear as Type $A$ assignments. They have been classified by their main characteristic hypothes is $(\mathrm{MH})$ and their object, obstacle or weather object hypothesis $(\mathrm{OH})$ [8].

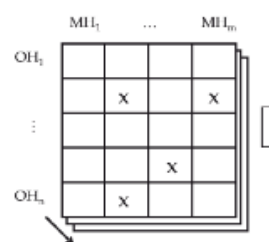

Generation

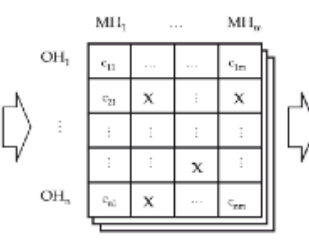

Determination

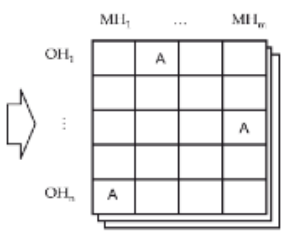

Selection
Fig. 10 Sensor data fusion

\section{EVALUATION}

The evaluation was carried out using a BMW research car, equipped with a full instrumentation for autonomous driving. The car is illustrated in fig. 11.

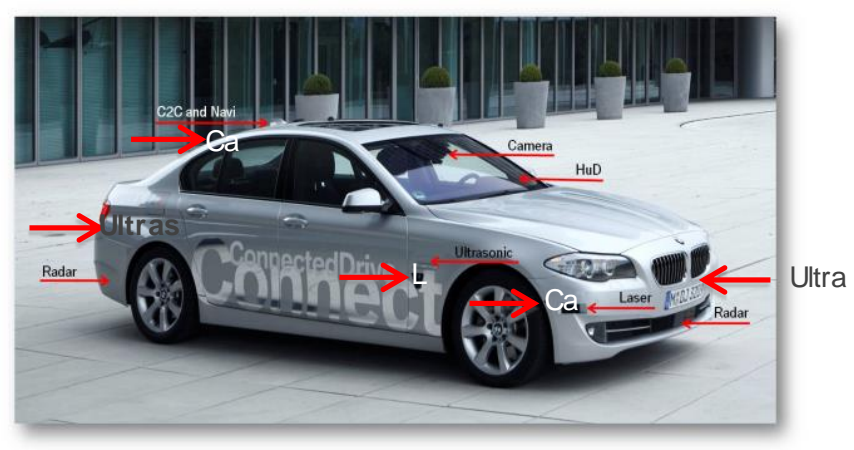

Fig. 11 Research car

Arrows mark the main sensors; in particular the LIDAR scanners are highlighted as lasers. For the different types of eco-data (air pollution and fog) conditions, particular test runs have been executed. Input and output has been captured for further studies to optimize the recognition algorithms. The outcome of the near field environmental (eco-) recognition is valuable eco-driving and drivingsecurity information for the driver. Additionally, such ecological data can be correlated with the current geo position (map location) and submitted to eco-telematics 
services or other collaborative traffic systems. On board of the vehicle itself many autonomous driving functions can subscribe these eco information's. Thus the adaptive cruise control (ACC) can automatically reduce the desired speed in case the eco conditions are getting worse. The same applies for highly automated driving. The recommended maximum speed setting, the driving attitude or the proposed routing can be adjusted automatically too.

\section{CONCLUSION}

Increasingly the automotive industry is developing and producing innovative driving as sistance functions to support vehicle drivers. Well understood as being a very important goal is to improve the eco driving ability. In this context it is an essential functionality to capture local eco-data (airpollution, fog or even weather) in the vicinity of the car. Many dependent functions within a vehicle can be advantaged by subscribing to an automated, reliable ecodata source. Focusing on the ecological and collaborative aspects of traffic systems existing today and as planned for the future will accelerate the process of producing eco friendly and reliable autonomous driving systems. It is clear that the detection of eco-data (and thus, road conditions) is a principal element and data source in this quest. Sharing information from vehicles with the sensors described in this paper is regarded as an intrinsic benefit for future green eco driving and so is being investigated for viability as part of our research work. Cloud based technologies are thought to be key for resolving the data communications aspects of this scenario, with traffic management and eco-data information systems interacting at the data level in a Cloud technology space. In our scenario, the data and software in this source would provide both driver information and vehicle control system data relating to vehicle position on the road, speed and road conditions, supplemented by eco-data such as air pollution and fog condition information to minimize the emissions of greenhouse gases.

\section{REFERENCES}

[1] Mario Pavlic, Heidrun Belzner, Gerhard Rigoll and Slobodan Ilic, "Image based fog detection in vehicles" Intelligent Vehicles Symposium (IV), 2012 IEEE, IEEE Press, June 2012, pp. 1132 1137, doi: 10.1 109/IVS.2012.6232256.

[2] Aubert, Didier, et al. "Digital imaging for assessing and improving highway visibility." TRA-Transportation Research Arena(2014).

[3] LEONARD, John, et al. A perception- driven autonomous urban vehicle. Journal of Field Robotics, 2008, 25. Jg., Nr. 10, S. 727-774.

[4] UTKIN, A. B., et al. Forest-fire detection by means of lidar. In: Proceedings of IV International Conference on Forest Fire Research, Luso, Portugal. 2002. S. 58.

[5] HSU, Chan Wei; HSU, Tsung Hua; CHANG, Kuang Jen. Implementation of car-following system using LiDAR detection. In: IT S Telecommunications (IT ST), 2012 12th International Conference on. IEEE, 2012. S. 165-169.

[6] DANIELS, Michele K., et al. Comparison of lidar-based and radarbased adaptive cruise control systems. SAE International, 2000.

[7] WERNER, Christian, et al. Visibility and cloud lidar. Springer New York, 2005.

[8] WINNER, Hermann; HAKULI, Stephan; WOLF, Gabriele (Hg.). Handbuch Fahrerassistenzsysteme. Springer DE, 2011.

[9] Bluesky International Limited. "How LIDAR works" http://www.lidar-uk.com/how-lidar-works/ 